\title{
More sideshows for solar neutrinos
}

\author{
It seems agreed that the numbers of detectable neutrinos emitted by the Sun are smaller than they \\ should be, but people are still vainly searching for an explanation.
}

THE matter of the missing neutrinos from the Sun becomes ever-more tortuous. It is now well over a decade since R. Davis's experiment, deep underground in the Homestake mine in the United States, first revealed that there are only a third as many neutrinos emitted from the Sun as the supposedly well-understood nuclear physics of the energy-producing core would suggest there should be.

Since then, the chief consequence has been that speculation about the origin of the discrepancy has flourished. But it seems to be generally agreed that further experiments will be needed to tell whether the discrepancy arises because knowledge of the interior of the Sun is inadequate or because neutrinos and their interaction with matter are not fully understood.

Davis's experiment, it will be recalled, consists of a large tank full of tetrachloroethylene (once used for removing grease from people's clothes). Although neutrinos interact only weakly with most particles of matter, they will occasionally convert nuclei of ${ }^{37} \mathrm{Cl}$ into nuclei of ${ }^{37} \mathrm{Ar}$, which is radioactive and thus detectable in small numbers.

Davis and his colleagues were at the outset confident of their finding that the rate of conversion of ${ }^{37} \mathrm{Cl}$ into ${ }^{37} \mathrm{Ar}$ is roughly a third of what it would be in the light of the expected flux of neutrinos from the Sun. Neither further examination nor the passage of time has seen them waver in this conviction.

Now there has arisen a further source of distraction in a field already sufficiently confused - the possibility that some of the conversion of chlorine to argon nuclei observed originally by Davis may be driven not by neutrinos from the core of the Sun, but by solar flares. The suggestion appears to have been made last year by Davis himself, based on an apparent correlation between records of the Homestake equipment and the presence of flares on the Sun. What seems to have weighed most with Davis is that an experimental run begun during 1972 spanned the occurence of the great solar flare of August that year and happened to be the run in which chlorine conversion (to argon) was most abundant.

Evidently, if this speculation were correct, the discrepancy between the expected and measured fluxes of neutrinos from the Sun would be further magnified. For the predicted numbers are those expected to be generated by nuclear reac- tions in the energy-producing core. If flares might be the source of some of the observed neutrinos, the core must be even less prolific a source than had been supposed.

Luckily, but by means of climax followed by anticlimax (Phys. Rev. Lett. $61,2650 \& 2652 ; 1988)$ this spectre has now apparently been laid. First, John D. Bahall from the Institute for Advanced Study at Princeton argues that if the Homstake detector is able to record neutrinos from solar flares, other neutrino detectors now in operation or construction should be even more drastically affected by them.

But how can a mere solar flare perform as if it were material in the high-temperature core of the Sun? One possibility is that nuclear collisions may generate both pions and muons which, when they decay, yield neutrinos detectable at Homstake. The trouble, says Bahcall, is that even the great flare of 1972 would have generated too few of them to be detectable at Homestake in the numbers reported by Davis. On similar grounds, he also rejects the notion that solar flares may influence the Homestake experiment by changing (reducing) the flux of more distant cosmic rays reaching the Earth and thus, indirectly, the amount of artificial radioactivity generated in the high atmosphere, which might indirectly affect the experiment.

But if there is some means by which mesons produced in solar flares can influence the conversion of ${ }^{37} \mathrm{Cl}$ into ${ }^{37} \mathrm{Ar}$, Bahcall's argument goes, then the other neutrino detectors either built or planned should be even more sensitively affected. Obligingly, he calculates the sensitivity of the two principal detectors now in operation, the Japanese instrument (whose detector is built around 2,000 tonnes of water) called Kamiokande and the IrvineMichigan-Brookhaven detector (IMB for short), with three times as much water

Each instrument is designed so that the interaction between a neutrino and a nuc leon or electron can be followed as if it were a regular measurement in highenergy physics. Both detectors are now famous for having recorded neutrinos from the supernova event of February, 1987 (11 and 8 events respectively). Bahcall quite rightly says that there should soon be a chance to settle the question whether solar flares can be a source of neutrinos, for is there not a solar maximum on the way?
Solar neutrino physicists, mercifully, will not have to wait even that long. A group of 35 physicists, mostly Japanese but with a strong group from the University of Pennsylvania, report in a succeeding article what amounts to their failure to associate the detection of neutrinos in the Kamiokande tank of water with the occurrence of solar flares. One of the interesting features of the Kamiokande measurements is that it has been possible to use as measures of the intensity of solar flares occurring sporadically not just their visual appearence, or magnetic effects on the surface of the Earth, but the intensity of the proton flux within them as measured by satellites in orbit for this among other purposes.

In all cases, it seems, the measurements at Kamiokande are insignificantly different from the background flux at times of solar flares or even the appearance of $\gamma$-ray flux in the galactic cosmic rays. The undramatic but comforting conclusion is that Davis's original discrepancy is not worsened by the suspicion that solar flares somehow play a part in the detection of neutrinos on the surface of the Earth.

Quite what that means for Davis's report last year that there is a correlation remains to be determined. On the face of things, there is no reason why his directconversion experiment should be affected by solar flares more than are measurements of the high-energy physics type in Japan and at IMB. But, in a field in which there have already been many surprises, nobody should count on that

The obvious and long-standing difficulty remains that the solar neutrinos measured by ${ }^{37} \mathrm{Cl}$ conversion span a range of energy different from that to which other detectors would be sensitive (whence Bahcall's scheme for using virtually the whole world's stock of gallium as a detector sensitive in a different energy range). But the possibility also remains that the neutrinos generated in the interior of the Sun are somehow modified in their traverse of the huge envelope around the core, most simply by the conversion of one type of neutrino (the electron-neutrino) into one of the other two (associated with muons and tauons, the heavier versions of the electron). However this tale comes out, it will remain a marvel that so much work, experimental as well as theoretical, has been stimulated by a single discrepant observation.

John Maddox 